\title{
A CONVENIENT MASKING GROUP FOR ARYL IODIDES
}

\author{
Jeffrey S. Moore*, Edward J. Weinstein, and Ziyan Wu \\ The Willard H. Dow Laboratories, Department of Chemistry, The University of Michigan \\ Ann Arbor, MI 48109-1055
}

SUMMARY: 1-Aryl-3,3-dialkyltriazenes give excellent yields of easily isolated aryl iodides upon treatment with methyl iodide.

Haloarenes often play an important role in the preparation of substituted aromatic compounds, and thus efficient means of masking the aryl halide bond are synthetically valuable. The ideal masking group would be readily available, stable to a variety of chemical conversions, and conveniently transformed under mild conditions and in high yield to the desired aryl halide. In light of the enhanced reactivity of aryl iodides (e.g. in transition-metal-catalyzed cross-coupling chemistry ${ }^{1}$ and in the direct preparation of arylzinc reagents ${ }^{2}$ ) a masking group for this particular haloarene would be especially useful. Unfortunately, few of the known aryl iodide syntheses meet the above-mentioned criteria. Barrio and co-workers, however, have reported on the conversion of 1-aryl-3,3-dialkyltriazenes to aryl iodides using either trimethylsilyl iodide ${ }^{3}$ or $\mathrm{NaI}$ with a cationic exchange resin ${ }^{4}$ in acetonitrile. In exploring the triazene group as a possible aryl iodide mask, we discovered a convenient and mild procedure for preparing aryl iodides from triazenes in excellent yield under neutral conditions. We report below the results of these investigations.

Treating 1-aryl-3,3-dialkyltriazenes with methyl iodide at reflux for several days gave only trace quantities of aryl iodide and unrecovered starting material. We then discovered that when this reaction was run in a sealed tube at $100-120^{\circ} \mathrm{C}$, nearly quantitative formation of the aryl iodide occurred in under $6 \mathrm{~h}$ (eq 1). No trace of other side products could be detected by GC.5 We then examined the reaction in

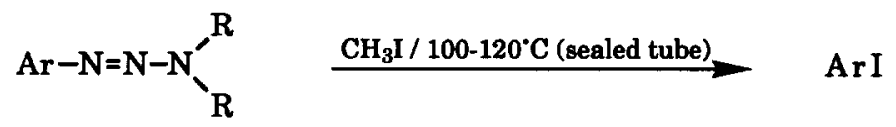

more detail as summarized in Table I. These conditions proved to be quite general, giving excellent yields of easily isolated products. As seen in Table I, these conditions are suitable for a variety of functional groups including trimethylsilyl acetylenes which we were unable to obtain in reasonable yields using the conditions reported by Barrio and co-workers. ${ }^{3,4}$ Since triazenes are readily available from aromatic amines, $3,4,6$ and stable to a variety of synthetic conditions, ${ }^{7}$ this group serves as an efficient mask for the aryl iodide bond.

While yields tended to generally be quite high, we found the reaction rate to be somewhat dependent on para-aromatic substituents as indicated in Table $I$. The rate differences observed for the various substituted aromatics, however, are not so severe as to detract from the synthetic usefulness of this reaction. We have also performed the reaction in benzene using 5 equiv of methyl iodide. Under these 
conditions excellent yields are still obtained although longer reaction times are required. Finally, it can be seen that the reaction can be performed with equal success using various N,N-dialkyl substituents. 8

A representative experimental procedure is as follows. 1-(4-Bromophenyl)-3,3-diethyltriazene ( 320 $\mathrm{mg}, 1.25 \mathrm{mmol}$ ) was taken up in freshly distilled methyl iodide $(\sim 5 \mathrm{~mL})$. The solution was degassed, placed under a nitrogen head space, and the tube sealed. ${ }^{9}$ After heating for $6 \mathrm{~h}$ at $120^{\circ} \mathrm{C}$, the starting triazene had been completely consumed. During this time, diethyldimethyl ammonium iodide precipitated from solution. The reaction mixture was poured into petroleum ether $(25 \mathrm{~mL})$, filtered, and concentrated. Following filtration through a short plug of silica gel, pure 4-iodobromobenzene was obtained. ${ }^{10}$ We have also routinely carried out this reaction on a 5-10 $\mathrm{g}$ scale in a Fischer-Porter bottle with equal success.

Acknowledgment is made to the Donors of the Petroleum Research Fund, administered by the American Chemical Society, for partial support of this work.

Table I

Triazene to Aryl Iodide Conversion

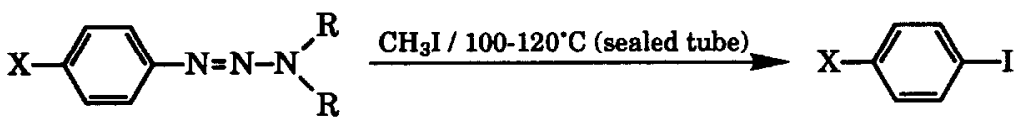

\begin{tabular}{c|c|c|c}
\hline$-\mathrm{X}$ & $\mathrm{R}$ & isolated yield (\%) & $\mathrm{k}_{\mathrm{rel}}$ \\
\hline$-\mathrm{H}$ & $\mathrm{C}_{2} \mathrm{H}_{5-}$ & $>95^{\mathrm{a}}$ & 1.0 \\
$-\mathrm{H}$ & $\mathrm{CH}_{3-}$ & $>95^{\mathrm{a}}$ & 1.0 \\
$-\mathrm{H}$ & $\left.-\mathrm{CH}_{2}\right)_{4}^{-} \mathrm{b}$ & $>95^{\mathrm{a}}$ & 1.1 \\
$-\mathrm{H}$ & $\mathrm{C}_{2} \mathrm{H}_{5-}$ & $>95^{\mathrm{a}, c}$ & -- \\
$-\mathrm{Br}$ & $\mathrm{C}_{2} \mathrm{H}_{5-}$ & 94 & .17 \\
$-\mathrm{OMe}$ & $\mathrm{C}_{2} \mathrm{H}_{5-}$ & 99 & 2.1 \\
$-\mathrm{CO}_{2} \mathrm{Me}$ & $\mathrm{C}_{2} \mathrm{H}_{5-}^{-}$ & 97 & $<0.1$ \\
$-\mathrm{OAc}^{-}$ & $\mathrm{C}_{2} \mathrm{H}_{5-}$ & 95 & -- \\
$-\mathrm{C} \equiv \mathrm{C}-\mathrm{SiMe}_{3}$ & $\mathrm{C}_{2} \mathrm{H}_{5-}$ & 96 & --
\end{tabular}

aYield determined by gas chromatography using an internal standard.

byrrolidine

c Reaction carried out in benzene with 5 equiv of $\mathrm{CH}_{3} \mathrm{I}$.

1. See for example Trost, B. M.; Verhoeven, T. R. In Comprehensive Organometallic Chemistry; Wilkinson, G.; Stone, G. A.; Abel, E. W., Eds.; Pergamon: New York, NY, 1982; Vol. 8 p 913.

2. Majid, T. N.; Knochel, P. Tetrahedron Lett. 1990, $31,4413$.

3. H. Ku and J. R. Barrio J. Org. Chem. 1981, 46, 5239.

4. N. Satyamurthy and J. R. Barrio J. Org. Chem. 1983, 48, 4394.

5. By-products from the reaction were nitrogen gas and diethyldimethyl ammonium iodide, which was formed by alkylating the eliminated diethylmethyl amine.

6. See for example, L. Lunnazi, G. Cerioni, E. Foresti, and D. Macciantelli J. Chem. Soc., Perkin Trans. $1978,686$.

7. We are currently investigating the scope and limitations of 1-aryl-3,3-dialkyl triazenes with regard to stability under various synthetic conditions. Preliminary data indicate that the group is completely stable to standard conditions used in palladium-calalyzed coupling reactions.

8. We have prepared several pyrrolidine triazenes and found that these derivatives have a greater tendency to form crystalline solids than the corresponding N,N-diethyl or N,N-dimethyl derivatives. Thus, the pyrrolidine triazenes can easily be purified by recrystallization.

9. As a reusable, sealed-vessel for small-scale reactions, we found that heavy-walled glass tubing joined to a Teflon screwvalve was convenient.

10. All compounds were characterized by ${ }^{1} \mathrm{H}$ NMR ( $\left.300 \mathrm{MHz}\right), \mathrm{GC}$, and TLC. 